\title{
Structure of unital 3-fields
}

\author{
Steven Duplij • Wend Werner
}

Received: 14 January 2019 / Accepted: 27 July 2020 / Published online: 8 October 2020

(C) The Author(s) 2020

\begin{abstract}
We investigate fields in which addition requires three summands. These ternary fields are shown to be isomorphic to the set of invertible elements in a local ring $\mathcal{R}$ having $\mathbb{Z} / 2 \mathbb{Z}$ as a residual field. One of the important technical ingredients is to intrinsically characterize the maximal ideal of $\mathcal{R}$. We include a number of illustrative examples and prove that the structure of a finite 3-field is not connected to any binary field.
\end{abstract}

\section{Introduction}

Most of us seem to be biologically biased towards thinking that it always requires two in order to generate a third. In mathematics or physics, however, this idea does not seem to rest on a sound foundation: The theory of symmetric spaces, for example, is nicely described in terms of Lie or Jordan triple systems ([5, 20]; see e.g. [2] for a recent development), and in physics, higher Lie algebras have come into focus in [18] (for later development see e.g. [9, 15]) and were e.g. applied to the theory of M2-branes in [1]. Ternary Hopf algebras were introduced and investigated in [11].

In order to illustrate why oftentimes (but not here), ternary algebraic structure does not introduce new aspects, let us digress somewhat and have a closer look at a simple example, commutative ternary groups.

\footnotetext{
S. Duplij

Center for Information Technology (WWU IT), Westfälische Wilhelms-Universität Münster, 48149 Münster, Germany
}

W. Werner $(\bowtie)$

Mathematisches Institut, Westfälische Wilhelms-Universität Münster,

Einsteinstrasse 62, 48149 Münster, Germany

E-Mail: wwerner@uni-muenster.de 
If $G$ is a set and $m: G^{3} \rightarrow G$ is a mapping, what properties should $m$ have in order that $G$ be called a (commutative) ternary group with multiplication given by $m$ ? Here is a list.

- Associativity, as always, should mean that there is no need for brackets when we multiply several times in a row.

- The ternary multiplication is commutative, iff it is invariant under any permutation of the factors

- Finally, every element $g \in G$ has a (ternary) inverse $\bar{g}$ (the quer element) so that for all $g_{0} \in G$

$g_{0} g \bar{g}=g_{0}$.

This is all that is needed in the way of a definition. Note that there is no neutral element, which nonetheless can be defined by saying $e \in G$ is neutral, iff $e e g=g$, for all $g \in G$.

The reason that we did not include the existence of a neutral element $e$ in the definition is that as soon as there is one, $G$ equipped with the product

$$
g \times h:=g e h,
$$

becomes a binary group (with neutral element $e$ and inverses $g^{-1}=\bar{g}$ ) such that for all $g_{1,2,3} \in G$

$$
g_{1} \times g_{2} \times g_{3}=g_{1} g_{2} g_{3} .
$$

So here, $G$ is trivial in that its ternary product directly comes from a binary one. ${ }^{1}$ This, however always is the case, albeit for a different reason.

A more general example of this kind is the following. Pick a binary commutative group $G$, fix $g_{0} \in G$, and let

$$
m\left(g_{1}, g_{2}, g_{3}\right)=g_{0} g_{1} g_{2} g_{3}
$$

Then $(G, m)$ is a ternary group with $\bar{g}=g_{0}^{-1} g^{-1}$ for each $g \in G$. Also, $(G, m)$ is unital iff $g_{0}=w^{2}$ for some $w \in G$ in which case $e=w^{-1}$ is a unit.

No further examples exist, as we will see next. For a ternary, commutative group $G$ we consider the set of mappings

$$
P(G)=\left\{p_{a, b}: G \rightarrow G \mid a, b \in G, \quad p_{a, b}(g)=a b g\right\} .
$$

\footnotetext{
1 In binary parlance, units as defined here resemble reflections, and so no uniqueness of unit elements is to be expected. The resulting binary groups, though, are all pairwise isomorphic: If, in fact, $e_{1,2}$ are two units, and if we denote the corresponding binary groups by $G_{1}$ and $G_{2}$, repectively, then the mapping $\Phi: G_{1} \rightarrow G_{2}$, given by $\Phi(g)=e_{1} e_{2} g$ is easily seen to be an isomorphism.
} 
Under composition of mappings, $P(G)$ is a (binary) group (with unit $p_{g, \bar{g}}$, and $\left.p_{a, b}^{-1}=p_{\bar{a}, \bar{b}}\right)$. Fixing an element $g_{0} \in G$, we find that the map

$$
\Phi_{g_{0}}: G \rightarrow P(G), \quad \Phi_{g_{0}}(g)=p_{g, g_{0}}
$$

is a morphism of $G$ into $P(G)$, when the latter is equipped with the ternary product $p_{1} p_{2} p_{3}=p_{\bar{g}_{0}, \bar{g}_{0}} \circ p_{1} \circ p_{2} \circ p_{3}$, and we obtain

For any commutative ternary group $G$ there is a binary group $G_{0}$ as well as an element $g_{0} \in G_{0}$ such that $G$ is isomorphic to the ternary group defined on $G_{0}$ through the ternary product $m\left(g_{1}, g_{2}, g_{3}\right)=g_{0} g_{1} g_{2} g_{3}$.

And we conclude: As far as commutative ternary groups are concerned, the theory doesn't produce much of a novelty.

In this paper we investigate 3-fields, a structure in which the binary operations of the classical theory are replaced by ternary ones. There is a marked difference between addition and multiplication, due in principle to the different way in which addition and multiplication enter the distributive law. Whereas the multiplicative structure of higher arity in rings makes easier contact with binary algebra (see e.g. [17] or [12], for example) largely based on the fact that, as above, multiplicative pairs can be used in these examples just as we did here for commutative ternary groups, ternary addition produces phenomena of a more unusual kind and has been, to the knowledge of the authors, treated less thoroughly. We therefore keep multiplication in the fields binary for the moment and stick to ternary addition. Technically, this fact is hidden behind the expression unital, as in ternary group theory one can very well dispose of a unit, and, even more strikingly, the truly ternary case is characterized by the absence of one.

It turns out that there are a number (actually, one that might turn out to be too large) of interesting examples of 3-fields, finite ones, a certain subset of the 2-adic numbers, a class of finite skew-3-fields, based on the quaternion group, or a number of group 3-algebras which actually turn out to be 3-fields.

Here is what we will do in the following: The first section collects some basic theory (based on the pioneering papers $[10,19]$ ), Sect. 2 introduces the main technical tool that permits a connection to binary algebra, in the third section we deal with ideals which probably provide the most uncommon definition in this paper, Sect. 4 is brief on 3-vector spaces and 3-algebras, just enough in order to be well equipped for a first attack on the classification of finite 3 -fields in the final section. Among other things, we will prove here that the number of elements of a finite 3 -field is a power of two, that their structure is governed by certain polynomials, with coefficients from the unit disk $\mathcal{B}_{2}$ of the 2-adic number field $\mathbb{Q}_{2}$ and (in the case of a single generator, mapping $\partial \mathcal{B}_{2}$ into the interior of $\mathcal{B}_{2}$ ). Furthermore, each such field carries a structure totally different from classical fields, because essentially none of the finite ternary unital 3-fields embeds into a binary field, when the latter is supposed to carry its canonical ternary structure. 


\section{Some history, basics and examples}

The present topic has some precursors. Besides the ones mentioned in the previous section, groups with additional, quite general "multiple operators" were considered in abstract form in the late 50's and 60's (with [13] probably being a first reference for this perspective; see also the survey [16]). Our construction is connected with the notion of $(n, m)$-rings which was introduced in [8] and further studied in [4, 7]. The Post theorem for $(n, m)$-rings was formulated in [6]. In almost all of these investigations, the authors treat the case of $n$-fold sums and products; in many respects, though, the case where $n=3$, shows some more particular features so that we, for now, restrict our attention to this special case.

First we recall the general notion of a $(3,3)$-ring $[4,6]$. We have two different operations on a set $\mathcal{R}$ : the ternary addition $v: \mathcal{R} \times \mathcal{R} \times \mathcal{R} \rightarrow \mathcal{R}$ and the ternary multiplication $\mu: \mathcal{R} \times \mathcal{R} \times \mathcal{R} \rightarrow \mathcal{R}$. We suppose that both operations are totally associative

$$
\begin{aligned}
& v(v(x, y, z), t, u)=v(x, v(y, z, t), u)=v(x, y, v(z, t, u)), \\
& \mu(\mu(x, y, z), t, u)=\mu(x, \mu(y, z, t), u)=\mu(x, y, \mu(z, t, u)),
\end{aligned}
$$

where $x, y, z, t, u \in \mathcal{R}$. This means that both $\langle\mathcal{R}, v\rangle$ and $\langle\mathcal{R}, \mu\rangle$ are ternary semigroups. The connection between them is given by a ternary analog of the distributive law. A general form of the ternary distributivity (which we will use here) is

$$
\begin{aligned}
& \mu(v(x, y, z), t, u)=v(\mu(x, t, u), \mu(y, t, u), \mu(z, t, u)), \\
& \mu(t, v(x, y, z), u)=v(\mu(t, x, u), \mu(t, y, u), \mu(t, z, u)), \\
& \mu(t, u, v(x, y, z))=v(\mu(t, u, x), \mu(t, u, y), \mu(t, u, z)) .
\end{aligned}
$$

The semigroup $\langle\mathcal{R}, v\rangle$ is assumed to be a ternary group so that for all $a, b, c \in \mathcal{R}$ there exists a unique solution of the equation $[10,19]$,

$$
v(a, b, x)=c .
$$

Here, the important notion of a querelement [10], denoted by $\widetilde{x}$ for the addition $v$ comes into play. It satisfies

$$
v(y, x, \widetilde{x})=x,
$$

for all $y \in \mathcal{R}$. Dörnte shows in his paper that the existence of querelements for all $x \in \mathcal{R}$ is equivalent to unique solvability (6, his axiom $P_{3}$ ), and, in particular, the querelement is uniquely determined.

Definition 1 A set $\mathcal{R}$ with two operations $v$ and $\mu$ satisfying distributivity and for which $\langle\mathcal{R}, v\rangle$ is a (commutative) ternary group and $\langle\mathcal{R}, \mu\rangle$ is a ternary semigroup is called a $(3,3)$-ring, or for shortness, a 3-ring. 
Definition 2 If ternary multiplication $\mu$ on $\mathcal{R}$ is commutative, i.e. if $\mu=\mu \circ \sigma$, where $\sigma$ is any permutation from $S_{3}$, then we call $\mathcal{R}$ a commutative 3-ring.

Definition 3 An element 0 of a 3-ring $\mathcal{R}$, is called a ternary zero iff

$$
\mu(0, x, y)=\mu(x, 0, y)=\mu(x, y, 0)=0
$$

for all $x, y \in \mathcal{R}$.

Distributivity in $\mathcal{R}$ shows that $v(0,0, x)=x$ for all $x \in \mathcal{R}$. Furthermore, other than a mere neutral element for 3-group, a zero element of a 3-ring is uniquely determined.

Definition 4 Let $\mathcal{R}$ be a (3,3)-ring. $\mathcal{R}$ is called a (2,3)-ring, if its addition $v$ is derived from a binary addition + , i.e. $v(x, y, z)=x+y+z$. Similarly, it is called a $(3,2)$-ring, if its multiplication $\mu$ is derived from a binary multiplication $\cdot$, i.e. $\mu(x, y, z)=x \cdot y \cdot z$.

Proposition 1 Suppose that $\mathcal{R}$ is a $(3,3)$-ring.

1. Under the assumption that $\mathcal{R}$ contains a multiplicative unit 1 ,

$$
a \bullet b:=\mu(a, 1, b),
$$

yields a binary associative, commutative product so that $\mu(a, b, c)=a \bullet b \bullet c$, and $\mathcal{R}$ may be viewed as a $(3,2)$-ring.

2. If $\mathcal{R}$ contains a zero element 0 , then, similar as in the previous statement,

$$
a+b:=v(a, 0, b)
$$

defines a binary, associative and commutative composition with $v(a, b, c)=$ $a+b+c$, and $\mathcal{R}$ is $a(2,3)$-ring.

3. Whenever $\mathcal{R}$ contains both, 1 and 0 , then it is a binary ring.

The proof of this statement (which in large part is similar to the corresponding one for commutative ternary groups as in the introduction), which we do not want to present here, essentially consists of writing out all the involved definitions and observing that the above statements indeed are correct. Note, however, that it is important to have a zero elment in $\mathcal{R}$ (as opposed to a merely additive neutral element), as this is needed in order to show that the binary addition satisfies the distributive law.

Definition 5 We call $\mathcal{R}$ a proper (3,3)-ring, iff neither $\mu$ nor $v$ are derived, and $\mathcal{R}$ is a proper unital 3-ring, iff (only) its multiplication $v$ is derived.

We now come to the central defintion of this paper, the concept of a 3-field. Fields of this type seem to have first appeared in [7, Defintion 3.1] and [17, Defintion 3.5]. In these papers, a zero element $z$ is allowed to be contained in a commutative 3 -field (so that if a multiplicative unit exists, the binary situation is covered as well). The 
aim of the following is to focus on the less familiar situation and to require, right from the start, that no zero exists in a unital 3-field.

Definition 6 A 3-ring is called a 3-field if it is a group with respect to multiplication, and it is called unital if it contains a multiplicatively neutral element (so that its multiplicative group is derived).

Stated differently, a unital 3-field simultaneously carries the structure of a binary multiplicative and ternary additive group which have to cooperate through the distributive law. The reader should also note that the requirement that each element possesses a multiplicative inverse excludes the possibility that the underlying additive ternary group contains a zero element. It is possible, though, that there are neutral elements for the ternary additive group underlying a unital 3-field. The fields containing such an element are precisely the ones of characteristic 1 (see Proposition $3)$.

Example 1 The simplest example for a unital 3-field in which every element is additively neutral is $\{1, x\}$ where (necessarily) $x^{2}=1$. Note that it is only required here that one of these elements is additively neutral. Furthermore, there is only one more unitel 3 -field with 2 elements, the field $\{1,3\}$ inheriting its structure from $\mathbb{Z} / 4 \mathbb{Z}$ (see the example below).

Example 2 There are (2,3) fields without a (multiplicative) unit: $i \mathbb{R}$ becomes a $(2,3)$-field, when equipped with binary addition and multiplication inherited from the complex number field.

Example 3 The existence of a zero element in (3,3)-fields is not automatic, even if neutral elements abound: Let $\Phi_{2}=\{x, y\}$ and assume both elements are additively, as well as multiplicatively neutral. This yields the results of all possible ternary products and additions. It is also straightforward to check that the distributive law holds. But neither $x$ nor $y$ is a zero element for this field. Note that also any cartesian power of $\Phi_{2}$ is a $(3,3)$ field in which all elements are additively and multiplicatively neutral. Using the fundamental theorem of finitely generated abelian groups one can see that each field with this property necessarily has $2^{k}$ elements.

Example 4 For a more general construction, start with a unital 3-field F. Fix a unital 3-subfield $\mathbb{F}_{1}$ as well as an element $t \in \mathbb{F} \backslash \mathbb{F}_{1}$ so that $t^{2} \in \mathbb{F}_{1}$. Then $t \mathbb{F}_{1}$ is a $(3,3)$-field in which none of the algebraic operations is derived.

Example 5 A set of finite unital 3-fields is given by

$$
\left(\mathbb{Z} / 2^{n} \mathbb{Z}\right)^{\text {odd }}=\left\{2 k+1 \in \mathbb{Z} / 2^{n} \mathbb{Z} \mid 0 \leq 2 k+1 \leq 2^{n}\right\} .
$$

The fact that each element has a multiplicative inverse follows from the fact that $\operatorname{gcd}\left(a, 2^{n}\right)=1$, for all $a \in\left(\mathbb{Z} / 2^{n} \mathbb{Z}\right)^{\text {odd }}$. 
Recall that a cancellative and commutative 3 -ring $\mathcal{R}$ is called a 3 -integral domain [7].

Example 6 (3-field of fractions [7]) For any 3-integral domain in which neither ternary addition nor multiplication are derived the 3-field of fractions as defined in [7] is a proper 3-field. For instance, starting with

$$
\mathbb{Z}^{\text {odd }}=\{2 k+1 \mid k \in \mathbb{Z}\},
$$

we arrive at the proper 3 -field

$$
\mathbb{Q}^{\text {odd }}=\left\{r \in \mathbb{Q} \mid \exists p, q \in \mathbb{Z}^{\text {odd }}, r=\frac{p}{q}\right\} .
$$

Trying to find a completion of $\mathbb{Q}^{\text {odd }}$ which itself is a proper 3-field one has to avoid a zero element in the process. The easiest way to do this seems to be to exploit the relationship of $\mathbb{Q}^{\text {odd }}$ with the field of dyadic numbers, $\mathbb{Q}_{2}$. Recall the definition of the absolute value $|\cdot|_{2}$. If $\frac{p}{q}=2^{r} \frac{p_{0}}{q_{0}}$, where neither of the integers $p_{0}$ and $q_{0}$ is divisible by 2 , we have

$$
\left|\frac{p}{q}\right|_{2}=2^{-r} \text {. }
$$

Completion of $\mathbb{Q}$ w.r.t. $|\cdot|_{2}$ results in the field $\mathbb{Q}_{2}$, the elements of which can be formally written as

$$
x=\sum_{r \geq-n_{0}} \varepsilon_{r} 2^{r}, \quad \varepsilon_{r} \in \mathbb{Z} / 2 \mathbb{Z} .
$$

and $|x|_{2}=2^{n_{0}}$. Then $|x|_{2}=1$, iff

$$
x=1+\sum_{r=1}^{\infty} \varepsilon_{r} 2^{r}, \quad \varepsilon_{r} \in \mathbb{Z} / 2 \mathbb{Z} .
$$

Example 7 The set $\mathbb{Q}_{2}^{\text {odd }}=\left\{\left.x \in \mathbb{Q}_{2}|| x\right|_{2}=1\right\}$ is a unital 3-field w.r.t multiplication and ternary addition inherited from $\mathbb{Q}_{2}$. This field is the completion of $\mathbb{Q}^{\text {odd }}$ w.r.t. $|\cdot|_{2}$. Note that this 3 -field is compact. Furthermore, similar to the binary case, $\mathbb{Q}_{2}^{\text {odd }}$ is an inverse limit $\mathbb{Q}_{2}^{\text {odd }}=\lim _{\longleftarrow}\left(\mathbb{Z} / 2^{n} \mathbb{Z}\right)^{\text {odd }}$.

\section{Pairs}

One of the striking differences of the ternary in comparison to to the binary theory is the fact that for 3-fields there exists a meaningful ideal theory (which will be initiated in the following section). Central to this concept in classical theories is 
the 1-1 correspondence between ideals and kernels of morphisms, a relationship seemingly doomed in the absence of a zero element.

The purpose of the present section is to find a zero element for a unital 3-field $\mathbb{F}$, not too far away from $\mathbb{F}$, by embedding it into a binary unitary ring, in an essentially unique way, and carefully enough so that the essential structure of $\mathbb{F}$ is preserved.

The starting point is the following definition in which we will strip down the notation and use plus signs even at places where we deal with a ternary addition. As it will turn out, this might be justified by the fact that the ternary addition of any 3 -field actually is induced by the binary addition of a canonical binary ring.

Definition 7 Let $\mathcal{R}$ be a unital 3-ring, and for $a, b \in \mathcal{R}$ let

$$
q_{a, b}: \mathcal{R} \rightarrow \mathcal{R}, \quad x \longrightarrow x+a+b
$$

We will furthernmore use the notation

$$
\mathcal{Q}(\mathcal{R})=\left\{q_{a, b} \mid a, b \in \mathcal{R}\right\} \quad \text { and } \quad \mathcal{U}(\mathcal{R})=\mathcal{Q}(\mathcal{R}) \cup \mathcal{R}
$$

Note that the above definition yields an equivalence relation on the Cartesian product $\mathcal{R} \times \mathcal{R}$. Similar constructions go back at least as far as [19], serving to "reduce arity".

First, we convert the set of pairs into a (binary) ring. In order to reduce the technical effort we write pairs in their standardforms: For each pair we have

$$
q_{a, b}=q_{a+b-1,1},
$$

and whenever $q_{s, 1}=q_{t, 1}$ then $s=t$. With this notation we (well-)define binary addition $+_{q}$ and the binary product $\times_{q}$ for pairs through

$$
\begin{aligned}
& q_{\alpha, 1}+{ }_{q} q_{\beta, 1}=q_{\alpha+\beta+1,1}, \\
& q_{\alpha, 1} \times{ }_{q} q_{\beta, 1}=q_{\alpha+\beta+\alpha \beta, 1} .
\end{aligned}
$$

We extend these operations to $\mathcal{U}(\mathcal{R})$. For $u, v \in \mathcal{U}(\mathcal{R})$ let

$$
\begin{aligned}
& u+\mathcal{u} v=\left\{\begin{aligned}
q_{u, v}, & u, v & \in \mathcal{R}, \\
u(v)=a+b+v, & u=q_{a, b} & \in \mathcal{Q}(\mathcal{R}), v \in \mathcal{R}, \\
u+{ }_{q} v, & u, v & \in \mathcal{Q}(\mathcal{R}),
\end{aligned}\right. \\
& u \times_{\mathcal{U}} v=\left\{\begin{array}{cc}
u v, & u, v \in \mathcal{R}, \\
q_{a v, b v}, & u \in \mathcal{Q}(\mathcal{R}), v \in \mathcal{R}, \\
u \times_{q} v, & u, v \in \mathcal{Q}(\mathcal{R}),
\end{array}\right.
\end{aligned}
$$

These operations are well-defined and we furthermore have

Theorem $1\left\langle\mathcal{Q}(\mathcal{R}),+_{q}, \times_{q}\right\rangle$ and $\left\langle\mathcal{U}(\mathcal{R}),+_{u}, \times_{u}\right\rangle$ are binary rings, $\mathcal{U}(\mathcal{R})$ is unital of which $\mathcal{R}$ is a subring, whereas $\mathcal{Q}(\mathcal{R})$ is an ideal. 
For the somewhat lengthy proof it is very convenient to use pairs in their standard forms, and then there are no major obstacles, although, due to the definition of the algebraic operations, quite a number different cases have to be distinguished.

Example $8 \quad$ Let $\mathcal{R}=\mathbb{Z}^{\text {odd }}$. Then $\varphi: \mathcal{Q}(\mathcal{R}) \rightarrow \mathbb{Z}^{\text {even }}, q_{a, b} \stackrel{\varphi}{\longmapsto} a+b$, is a welldefined isomorphism of binary (nonunital) rings, and $\mathcal{U}(\mathcal{R})$ equals $\mathbb{Z}$. Similarly, for the unital 3-field $\mathbb{Q}^{\text {odd }}$ we have

$$
\mathcal{Q}\left(\mathbb{Q}^{\text {odd }}\right)=\mathbb{Q}^{\text {even }}=\left\{r \in \mathbb{Q} \mid \exists p \in \mathbb{Z}^{\text {even }}, q \in \mathbb{Z}^{\text {odd }}, r=\frac{p}{q}\right\},
$$

as well as

$$
\mathcal{U}\left(\mathbb{Q}^{\text {odd }}\right)=\mathbb{Q}^{\text {even }} \cup \mathbb{Q}^{\text {odd }}=\left\{r \in \mathbb{Q} \mid \exists p \in \mathbb{Z}, q \in \mathbb{Z}^{\text {odd }}, r=\frac{p}{q}\right\} .
$$

In the same vein,

$$
\mathcal{Q}\left(\left(\mathbb{Z} / 2^{n} \mathbb{Z}\right)^{\text {odd }}\right)=\left(\mathbb{Z} / 2^{n} \mathbb{Z}\right)^{\text {even }}, \quad \mathcal{U}\left(\left(\mathbb{Z} / 2^{n} \mathbb{Z}\right)^{\text {odd }}\right)=\left(\mathbb{Z} / 2^{n} \mathbb{Z}\right) .
$$

For the following observations it is convenient to use categorical language. We denote by $\mathfrak{F}_{3}$ the category in which the objects are unital 3-fields and where the morphisms are given by mappings between those which respect the additive and the multiplicative structure as well as the unit element. For each morphism between unital 3-fields $\phi: \mathbb{F}^{(1)} \rightarrow \mathbb{F}^{(2)}$ define mappings $\mathcal{Q} \phi: \mathcal{Q} \mathbb{F}^{(1)} \rightarrow \mathcal{Q F}{ }^{(2)}$ and $\mathcal{U} \phi:$ $\mathcal{U} \mathbb{F}^{(1)} \rightarrow \mathcal{U} \mathbb{F}^{(2)}$, by

$$
\begin{aligned}
& \mathcal{Q} \phi\left(q_{a, b}\right)=q_{\phi(a), \phi(b),} \\
& \mathcal{U} \phi(u)=\left\{\begin{array}{cl}
\mathcal{Q} \phi(u), & u \in \mathcal{Q} \mathbb{F}^{(1)}, \\
\phi(u), & u \in \mathbb{F}^{(1)}
\end{array}\right.
\end{aligned}
$$

It then requires only to invoke the involved defintions to see that $\mathcal{Q} \phi$ and $\mathcal{U} \phi$ are unital morphisms (with respect to binary addition and multiplication defined on these rings) so that we have defined a functor $\mathcal{U}$ from the category $\mathfrak{F}_{3}$ to the category of binary unital rings $\mathfrak{R}_{2}$.

Our next goal is to show that the embedding of a unital ternary field $F$ into $\mathcal{U}(F)$ is essentially the only way of embedding it into a binary unital ringt, in the following sense.

Theorem 2 (Universality theorem) Suppose $\mathcal{R}$ is a unital binary ring, equipped with the induced structure of a ternary unital ring, $\mathbb{F}$ is a unital 3-field, $\varphi: \mathbb{F} \rightarrow \mathcal{R}$ is a morphism of unital 3-rings, and write $i_{F}$ for the embedding $\mathbb{F} \rightarrow \mathcal{U}(\mathbb{F})$.

Then, there exists a morphism $\bar{\varphi}: \mathcal{U}(\mathbb{F}) \rightarrow \mathcal{R}$ of binary rings, such that $\bar{\varphi} \circ i_{F}=$ $\varphi$. 
Proof Define for $\mathcal{R}$ (which was supposed to carry its induced unital 3-ring structure) a map $\pi_{\mathcal{R}}: U(\mathcal{R}) \rightarrow \mathcal{R}$ by $\pi_{\mathcal{R}}(s)=r$ if $s=r \in \mathcal{R}$ and $\pi_{\mathcal{R}}(s)=1+a$ if $s=q_{1, a} \in \mathcal{Q}(\mathcal{R})$. Then it is straightforward to check that $\pi_{\mathcal{R}}$ is a unital ring morphism. ${ }^{2}$ If we now put $\bar{\varphi}=\pi_{\mathcal{R}} U(\phi)$ it follows that $\bar{\varphi} \circ i_{F}=\varphi$.

In the next result we will have a closer look at those rings that arise as $\mathcal{U}(F)$, for a unital 3-field $F$. Recall that a (commutative, binary) ring is called a local ring iff it contains a unique maximal ideal ${ }^{3}$

\section{Theorem 3 (3-fields and local rings)}

1. Let $\mathcal{R}$ be a (unital) local binary ring with (unique) maximal ideal $\mathcal{J}$ so that $\mathcal{R} / \mathcal{J} \cong \mathbb{Z} / 2 \mathbb{Z}$. Then $\mathcal{R} \backslash \mathcal{J}$ is a unital 3-field.

2. For any unital 3-field $\mathbb{F}$, there exists a local binary ring $\mathcal{R}$ with residual field $\mathbb{Z} / 2 \mathbb{Z}$ such that $\mathbb{F} \cong \mathcal{R} \backslash \mathcal{J}$, where $\mathcal{J}$ is the maximal ideal of $\mathcal{R}$ and $\mathcal{R} \backslash \mathcal{J}$ carries the derived ternary structure inherited from $\mathcal{R}$.

\section{Proof}

1. Let $\pi_{\mathcal{J}}: \mathcal{R} \rightarrow \mathcal{R} / \mathcal{J}$ be the quotient map. Suppose $a_{1,2,3} \in \mathcal{R} \backslash \mathcal{J}$. Since $a \in \mathcal{R} \backslash \mathcal{J}$, iff $\pi_{\mathcal{J}}(a)$ is in $\mathbb{Z} / 2 \mathbb{Z}$, it follows that $a_{1}+a_{2}+a_{3} \in \mathcal{R} \backslash \mathcal{J}$. It is straightforward to check that $\mathcal{R} \backslash \mathcal{J}$ is an additive 3-group. Similarly, the product $a_{1} a_{2} \in \mathcal{R} \backslash \mathcal{J}$, and distributivity is satisfied. It remains to show that each $a \in \mathcal{R} \backslash \mathcal{J}$ has a multiplicative inverse. Suppose $a$ has no inverse, by Krull's theorem it is contained in a maximal ideal different from $\mathcal{J}$, thus contradicting the locality of $\mathcal{R}$.

2. Let $\mathbb{F}$ be a unital 3-field. By Theorem $1 \mathcal{U}(\mathbb{F})$ is a binary unital ring. We show that $\mathcal{Q}(\mathbb{F})$ is a unique maximal ideal with $\mathcal{U}(\mathbb{F}) / \mathcal{Q}(\mathbb{F})=\mathbb{Z} / 2 \mathbb{Z}$. Evidently, $\mathcal{Q}(\mathbb{F})$ is an ideal of $\mathcal{U}(\mathbb{F})$. As all elements in $\mathcal{U}(\mathbb{F}) \backslash \mathcal{Q}(\mathbb{F})$ are invertible, this ideal has to be maximal. By the same reason, $\mathcal{Q}(\mathbb{F})$ is the only maximal ideal. So $\mathcal{U}(\mathbb{F})$ is a local ring.

It remains to show that $\mathcal{U}(\mathbb{F}) / \mathcal{Q}(\mathbb{F})=\mathbb{Z} / 2 \mathbb{Z}$. Take $r \in \mathcal{U}(\mathbb{F})$. If $r \in \mathbb{F}$, then $r+\mathcal{Q}(\mathbb{F})=1+\mathcal{Q}(\mathbb{F})$, because $r+\bar{r}+1=1$, and therefore $r \sim 1$. If $r \in \mathcal{Q}(\mathbb{F})$, then, of course, $r+\mathcal{Q}(\mathbb{F})=0+\mathcal{Q}(\mathbb{F})$, i.e. $r \sim 0$. So there are only two equivalence classes and hence $\mathcal{U}(\mathbb{F}) / \mathcal{Q}(\mathbb{F})=\mathbb{Z} / 2 \mathbb{Z}$.

It is not difficult to see that the functor $\mathcal{U}$ actually establishes an equivalence of the categories of unital local binary rings with residual field $\mathbb{Z} / 2 \mathbb{Z}$ and the category of unital 3-fields.

\footnotetext{
2 This somewhat tedious proof consists in going through a number of cases; for example, if $s_{1,2}=$ $r_{1,2} \in \mathcal{R}$ then $\quad \pi_{\mathcal{R}}\left(s_{1}+s_{2}\right)=\pi_{\mathcal{R}}\left(q_{1, r_{1}+r_{2}-1}\right)=r_{1}+r_{2}=\pi_{\mathcal{R}}\left(s_{1}\right)+\pi_{\mathcal{R}}\left(s_{2}\right)$.

All other cases are checked similarly.

3 The name for these rings comes from one of their most prominent examples: Consider the set of continuous functions defined in the neighbourhood of a point $k$ of some compact space $K$. Identifying two functions that coincide on a neighbourhood of $k$, yields a ring (with structure inherited from the ring of continuous functions) in which (the equivalence class of) those functions vanishing at $k$ form a unique maximal ideal. See e.g. [14, p. 111] for more.
} 
Example 9 In the case of $\mathbb{Q}_{2}^{\text {odd }}$, its local ring is the valuation ring $\mathcal{O}\left(\mathbb{Q}_{2}\right)=$ $\left\{\left.z \in \mathbb{Q}_{2}|| z\right|_{2} \leq 1\right\}$ with (maximal) evaluation ideal $\mathcal{B}\left(\mathbb{Q}_{2}\right)=\left\{\left.z \in \mathbb{Q}_{2}|| z\right|_{2}<1\right\}$, and $\mathbb{Q}_{2}^{\text {odd }}=\mathcal{O}\left(\mathbb{Q}_{2}\right) \backslash \mathcal{B}\left(\mathbb{Q}_{2}\right)$.

Here is another application:

Theorem 4 For any unital 3-field $\mathbb{F}$ the following are equivalent.

1. There exists an embedding of $\mathbb{F}$ into a binary field $\mathbb{K}$, where the latter is supposed to carry its derived ternary structure.

2. $\mathcal{Q}(\mathbb{F})$ is an integral domain.

3 . For each $y \neq 1$ the equation

$x+y-x y=1$

has the only solution $x=1$.

Proof Writing down what it means for $\mathcal{Q}(\mathbb{F})$ to be an integral domain, using standard forms of pairs, the equivalence of (2) and (3) is easily seen. Suppose that $\mathcal{Q}(\mathbb{F})$ is an integral domain, and denote by $\mathbb{K}$ its field of quotients. Define

$$
\Psi: \mathbb{F} \rightarrow \mathbb{K}, \quad \Psi(x)=\frac{q_{x, x}}{q_{1,1}}
$$

Then $\Psi$ is injective as $\Psi(x)=\Phi(y)$ is equivalent to

$$
q_{x+x, x+x}=q_{x, x} q_{1,1}=q_{y, y} q_{1,1}=q_{y+y, y+y}
$$

which by how pairs were defined is the same as $x=y$. The map $\Psi$ is also multiplicative, because $\Psi(x) \Psi(y)=\Psi(x y)$ is equivalent to

$$
q_{x y+x y, x y+x y} q_{1,1}=q_{x y, x y} q_{1+1,1+1}
$$

where on both sides the same pair is written in a slightly different form. Additivity is proven in a similar way.

Conversely, whenever there exists an embedding $\mathbb{F} \rightarrow \mathbb{K}, \mathcal{Q}(\mathbb{F})$ is injectively mapped into $\mathcal{Q}(\mathbb{K})$, which is an integral domain.

\section{Ideals}

Because of the absence of zero in a proper 3-ring, the usual correspondence between ideals and kernels of morphisms is no longer available. Instead, we apply the results of the previous section.

Let us consider a morphism of unital 3-rings $\phi: \mathcal{R}_{1} \rightarrow \mathcal{R}_{2}$. Then $\operatorname{ker} \mathcal{U}(\phi)$ is an ideal of $\mathcal{U}\left(\mathcal{R}_{1}\right)$, and the underlying equivalence relation on $\mathcal{R}_{1}$ is given by

$$
r_{1} \sim r_{2} \Longleftrightarrow \exists q \in \operatorname{ker} \mathcal{U}(\phi): r_{1}+q=r_{1}
$$


Note that $\operatorname{ker} \mathcal{U}(\phi)$ is contained in $\mathcal{Q}\left(\mathcal{R}_{1}\right)$, and so $q$ must be an additive pair. The above is a motivation for

Definition 8 An ideal for a unital 3-ring $\mathcal{R}$ is any (binary) ideal of $\mathcal{Q}(\mathcal{R})$. We furthermore denote the quotient an ideal of $\mathcal{Q}(\mathcal{R})$ defines on $\mathcal{R}$ by $\mathcal{R} / \mathcal{J}$.

Note that an ideal $\mathcal{J}$ intersecting $\mathcal{R}$ would lead to a zero element in the quotient $\mathcal{R} / \mathcal{J}$ and thus to a 3-ring whose additive structure is induced by a binary addition, a case that we are excluding here. Defining ideals in this way, the homomorphism theorem for 3-rings has a proof almost identical to the binary case.

Proposition 2 Suppose $\mathcal{R}$ and $\mathcal{S}$ are unital 3-rings, and $\phi: \mathcal{R} \rightarrow \mathcal{S}$ is a morphism. Then the quotient $\mathcal{R} / \operatorname{ker} \mathcal{U}(\phi)$ is a unital 3-ring, and $\mathcal{R} / \operatorname{ker} \mathcal{U}(\phi) \simeq \operatorname{Im} \phi$.

We prefer the expression "an ideal for a unital 3-ring" over "an ideal of ...", as the former is not a subset of $\mathcal{R}$.

The following theorem is an analogue to the fact that for a binary ring the quotient by an ideal is a field, if and only if the ideal is maximal.

Theorem 1 For a unital 3-ring $\mathcal{R}$ and an ideal $\mathcal{I} \subseteq \mathcal{Q}(\mathcal{R})$, the quotient $\mathcal{R} / \mathcal{I}$ is a unital 3-field, if and only if for any proper ideal $\mathcal{J}$ of $\mathcal{U}(\mathcal{R})$ for which $\mathcal{J} \supseteq \mathcal{I}$ it follows that $\mathcal{J} \cap \mathcal{R}=\varnothing$.

Proof Suppose that $\mathrm{F}=\mathcal{R} / \mathcal{I}$ is a unital 3-field but $\mathcal{J} \cap \mathcal{R} \neq \varnothing$ for some proper ideal $\mathcal{J}$ containing $\mathcal{I}$. Let $\pi: \mathcal{U}(\mathcal{R}) \rightarrow \mathcal{U}(\mathcal{R}) / \mathcal{I}$ be the quotient map. Note that there is a 1-1 correspondence between the ideals of $\mathcal{U}(\mathcal{R}) / \mathcal{I}$ and and the ideals of $\mathcal{U}(\mathcal{R})$ containing $\mathcal{I}$, given by

$$
\mathcal{J} \longrightarrow \pi(\mathcal{J}), \quad \text { resp. } \quad \mathcal{J}^{\prime} \longrightarrow \pi^{-1}\left(\mathcal{J}^{\prime}\right)
$$

(where $\pi \pi^{-1}\left(\mathcal{J}^{\prime}\right)=\mathcal{J}^{\prime}$ and $\pi^{-1} \pi(\mathcal{J})=\mathcal{J}$ ). It then follows that $\pi(\mathcal{J})$ is an ideal in $\mathcal{U}(\mathcal{R}) / \mathcal{I}=\mathcal{U}(\mathbb{F})$. Since $\varnothing \neq \pi(\mathcal{J} \cap \mathcal{R}) \subseteq \pi(\mathcal{J}) \cap \mathbb{F}$, this ideal contains an invertible element, hence $\pi(\mathcal{J})=\mathcal{U}(\mathbb{F})$, and so, again by the above correspondence, $\mathcal{J}=\mathcal{U}(\mathcal{R})$.

If, on the other hand, for any proper ideal $\mathcal{J} \supseteq \mathcal{I}$ we have $\mathcal{J} \cap \mathcal{R}=\varnothing$, we choose $r \in \mathcal{R} / \mathcal{I}$ as well as $r_{0} \in \mathcal{R}$ with $\pi\left(r_{0}\right)=r$. If $r$ were not invertible, then the ideal $\mathcal{J}_{0}$ generated by $r_{0}$ and $\mathcal{I}$ would be proper, contain $\mathcal{I}$ and intersect $\mathcal{R}$.

Example 10 Consider the (3,2)-ring $\mathbb{Z}^{\text {odd }}=\{2 k+1 \mid k \in \mathbb{Z}\}$. Note that each proper ideal in the non-unital ring $\mathbb{Z}^{\text {even }}$ is principal, i.e. it is of the form $\left(2 k_{0}\right)=$ $\left\{2 k_{0} k \mid k \in \mathbb{Z}\right\}, k_{0} \in \mathbb{Z}$. We claim that $\left(2 k_{0}\right)$ satisfies the hypothesis of Theorem 1 , if $k_{0}=2^{n}, n \in \mathbb{N}$. In fact, suppose that $p \mid k_{0}$ and $p \neq 2$. Then $(2 p) \supseteq \mathcal{I}$ and $p \in \mathbb{Z}^{\text {odd }}$, and so $\mathcal{I}$ cannot satisfy the maximiality condition from Theorem 1 .

Example 11 Let $\mathbb{F}$ be a proper unital 3-field, then each ideal in $\mathcal{U}(\mathbb{F})$ must be contained in $\mathcal{Q}(\mathbb{F})$ and has to fullfill the hypothesis of Theorem 1 (what could be called "evenly maximal" ), and so for each ideal $\mathcal{J}$ of $\mathcal{U}(\mathbb{F}), \mathbb{F} / \mathcal{J}$ again is a field. 
This is quite different from the binary case, where fields do not possess non-trivial quotients.

Example 12 The proper ideals for $\mathbb{Q}^{\text {odd }}=\left\{r \in \mathbb{Q} \mid \exists p, q \in \mathbb{Z}^{\text {odd }}, r=\frac{p}{q}\right\}$ are of the form

$$
\mathcal{J}_{n}=\left\{r \in \mathcal{U}\left(\mathbb{Q}^{\text {odd }}\right) \mid \exists q \in \mathbb{Z}^{\text {odd }}, \exists u \in \mathbb{Z}, r=2^{n} \frac{u}{q}\right\}=\left\langle 2^{n}\right\rangle, \quad n \in \mathbb{N}
$$

Obviously, all the $\mathcal{J}_{n}$ are ideals for $\mathbb{Q}^{\text {odd }}$. Conversely, let $\mathcal{J}$ be an ideal, and

$$
n_{0}=\min \left\{n \in \mathbb{N} \mid \exists p, q \in \mathbb{Z}^{\text {odd }}, 2^{n} \frac{p}{q} \in \mathcal{J}\right\} \text {. }
$$

Because any $r \in \mathcal{J}$ is of the form $2^{n} \frac{u}{q}, u \in \mathbb{Z}, q \in \mathbb{Z}^{\text {odd }}, n \geq n_{0}$ we must have $\mathcal{J}_{n_{0}} \supseteq \mathcal{J}$. Fix an element $2^{n_{0}} \frac{p_{0}}{q_{0}} \in \mathcal{J}, p_{0}, q_{0} \in \mathbb{Z}^{\text {odd }}$. Then $2^{n_{0}} \frac{u}{q} \in \mathcal{J}$ for all $u \in \mathbb{Z}$, $q \in \mathbb{Z}^{\text {odd }}$ and hence $\mathcal{J} \supseteq \mathcal{J}_{n_{0}}$.

We apply this observation to prime fields. Let us consider a unital 3-field $\mathbb{F}$ with unit 1 and define $\mathbb{F}^{\text {prim }}$ to be the 3 -subfield generated by 1 , i.e. $\mathbb{F}^{\text {prim }}=\langle 1\rangle$.

Definition 9 The characteristic of a unital 3-field is $\chi(\mathbb{F})=\left|\mathbb{F}^{\text {prim }}\right|$.

Theorem 2 If $\mathbb{F}^{\text {prim }}$ is finite, then there is $n \in \mathbb{N}_{0}$ so that $\mathbb{F}^{\text {prim }} \cong\left(\mathbb{Z} / 2^{n} \mathbb{Z}\right)^{\text {odd }}$. Otherwise, $\mathbb{F}^{\text {prim }} \cong \mathbb{Q}^{\text {odd }}$.

Proof Define a morphism $\psi: \mathbb{Q}^{\text {odd }} \rightarrow \mathbb{F}^{\text {prim }}$ by $\psi(p / q)=p q^{-1}$ which is welldefined and surjective (since $\operatorname{im} \psi$ is a 3-subfield containing 1), and so we must prove that $\mathbb{Q}^{\text {odd }} / \mathcal{J}_{n}=\mathbb{Z}_{2^{n}}^{\text {odd }}$, for $n \in \mathbb{N}_{0}$. Since the case $\operatorname{ker} \psi=\{0\}$ is trivial, we suppose $n>1$. Then division with reminder by $2^{n}$ yields a morphism $\mathbb{Z}^{\text {odd }} \rightarrow \mathbb{Z}_{2^{n}}^{\text {odd }}$, which extends to the quotient 3-field $\mathbb{Q}^{\text {odd }}$. It then follows that the kernel of this extension is the ideal $\mathcal{J}_{n}$.

We conclude this section with a characterisation of fields with characteristic 1 .

Proposition 3 A unital 3-fields $\mathbb{F}$ is of characteristic 1, if its additive 3-group contains a neutral element. 
Proof In a unital 3-field of characteristic 1, the unit always is a neutral element for the ternary addition (as is any other element). Conversely, if the element 1 is an additive neutral element then, for arbitrary elements $e, x \in \mathbb{F}$ we have

$$
x+e+e=e\left(e^{-1} x+1+1\right)=x,
$$

and hence, the characteristic of $\mathbb{F}$ equals one.

\section{3-vector spaces and unital 3-algebras}

We start by defining a ternary analogue of the concept of a vector space.

Definition 10 A 3-vector space consists of a commutative 3-group of vectors, $V$, a unital 3-field $\mathbb{F}$ as well as an action of $\mathbb{F}$ on $V$ (so hat $f(g v)=(f g) v$ for all $f, g \in \mathbb{F}$ and $v \in V$ ). Furthermore, $1 v=v$ for all $v \in V$, and the (ternary analog of the) standard distributivity laws hold. Linear mappings between 3-vector spaces are defined in the obvious way.

Given a 3-vector space $V$ over $\mathbb{F}$ we have a canonical action of $\mathcal{U}(\mathbb{F})$ on $\mathcal{U}(V)$, given in each of the as yet undefined cases $\mathbb{F} \times \mathcal{Q}(V) \rightarrow \mathcal{Q}(V), \mathcal{Q}(\mathbb{F}) \times V \rightarrow \mathcal{Q}(V)$, and $\mathcal{Q}(\mathbb{F}) \times \mathcal{Q}(V) \rightarrow \mathcal{Q}(V)$ similar to the definitions that led to Theorem 1 . With these actions in place, $\mathcal{U}(V)$ is a binary module over the binary ring $\mathcal{U}(\mathbb{F})$.

Definition 11 A subset $E \subseteq V$ of a 3-vector space over a unital 3-field $\mathbb{F}$ is called a generating system, if any element of $V$ can be represented as $\sum_{i=1}^{n} \lambda_{i} a_{i}$ with $\lambda_{i} \in U(\mathbb{F}), a_{i} \in E$, and $\sum_{i=1}^{n} \lambda_{i} \in \mathbb{F}$. A subset $A$ is called a basis, if this representation is unique. If $A$ is any subset of $V$ we denote by $\operatorname{lin} A$ the 3 -vector subspace of $V$ generated by $A$.

Remark 1 It is important to observe that any linear combination $\sum_{i=1}^{n} \lambda_{i} v_{i}$ with $\lambda_{i} \in U(\mathbb{F}), v_{i} \in V, \sum_{i=1}^{n} \lambda_{i} \in \mathbb{F}$ yields an element of $V$.

Example 13 A 3-vector space $V$ over the unital 3-field $\mathbb{F}$ is given by

$$
\left(\mathbb{F}^{n}\right)^{\text {free }}=\left\{\left(a_{1}, \ldots, a_{n}\right) \in \mathcal{U}(\mathbb{F}) \mid \sum_{i=1}^{n} a_{i} \in \mathbb{F}\right\} .
$$

It has a basis consisting of elements $e_{i}=\left(\delta_{i j}\right)_{j} \in\left(\mathbb{F}^{n}\right)^{\text {free }}$.

Example 14 Note that the Cartesian product $\mathbb{F}^{n}$ (which is a 3-field) is a 3-vector space as well, which however does not possess a basis if $n$ is different from 1 . If $n=1, \mathbb{F}^{\text {free }}=\mathbb{F}$, and any element of $\mathbb{F}$ is a basis.

Proposition 4 Every 3-vector space over a unital 3-field has a free resolution, i.e. there is a 3-vector space $V^{\text {free }}$ with basis which has $V$ as a quotient. 
Proof We pick a generating set $A$, and let $V^{\text {free }}=\left\{\sum_{\alpha \in A_{0} \text { finite }} f_{\alpha} \alpha \mid \sum f_{\alpha} \in \mathbb{F}\right\}$. Define $\phi_{V}: V^{\text {free }} \rightarrow V$ by $\sum_{\alpha \in A_{0} \text { finite }} f_{\alpha} \alpha \longmapsto \sum_{\alpha \in A_{0} \text { finite }} f_{\alpha} \alpha$ (so that the formal sum $\sum_{\alpha \in A_{0} \text { finite }} f_{\alpha} \alpha$ in $V^{\text {free }}$ is mapped to a sum in $V$ ). Then $\operatorname{ker} \phi_{V}$ is a $U(\mathbb{F})$ submodule contained in $\mathcal{Q}\left(V^{\text {free }}\right)$. Hence $V$ is isomorphic to $V^{\text {free }} / \operatorname{ker} \phi_{V}$.

Corollary 1 The number of elements of a 3-vector space over the finite 3-field $\mathbb{F}$, generated by $n$ elements is

$$
\frac{|\mathcal{U}(\mathbb{F})|^{n}}{2\left|\operatorname{ker} \phi_{V}\right|}=2^{n-1} \frac{|\mathbb{F}|^{n}}{\left|\operatorname{ker} \phi_{V}\right|}
$$

Proof We first note that the number of elements in the free 3-vector space of dimension $n$ is equal to half the number of elements in $\mathcal{U}(\mathbb{F})^{n}$ and so this statement follows from the above theorem as well as the fact that $|\mathcal{U}(\mathbb{F})|=2|\mathbb{F}|$ (which is rapidly seen by using the standard form of pairs).

Example 15 Consider $\mathbb{F}^{2}=\{1,3\}^{2}=\left\{\left(\begin{array}{c}a \\ b\end{array}\right) \mid a, b, \in(\mathbb{Z} / 4 \mathbb{Z})^{\text {odd }}\right\}$ over $\mathbb{F}=$ $(\mathbb{Z} / 4 \mathbb{Z})^{\text {odd }}=\{1,3\}$. We have $V=\left\{\left(\begin{array}{l}1 \\ 1\end{array}\right),\left(\begin{array}{l}3 \\ 1\end{array}\right),\left(\begin{array}{l}1 \\ 3\end{array}\right),\left(\begin{array}{l}3 \\ 3\end{array}\right)\right\}$. A generating set is $\left\{\left(\begin{array}{l}1 \\ 1\end{array}\right),\left(\begin{array}{l}3 \\ 1\end{array}\right)\right\}=\left\{e_{1}, e_{2}\right\}$. Thus, a free resolution is given by $V^{\text {free }}=$ $\left\{a e_{1}+b e_{2} \mid a, b \in \mathbb{Z} / 4 \mathbb{Z}, \quad a+b \in(\mathbb{Z} / 4 \mathbb{Z})^{\text {odd }}\right\}$.

Definition 12 Let $A$ be a 3-vector space over the unital 3-field $\mathbb{F}$. We call $A$ a unital (commutative) 3-algebra, if there exists a binary multiplication (•) on $A$ so that $(A,+, \bullet)$ is a (commutative) unital 3-ring.

In the following, 3-algebras will be mostly commutative.

Example 16 Let $G$ be a binary group and $\mathbb{F}$ a unital 3-field. The group algebra of $G$ over $\mathbb{F}$ is defined by

$$
\mathbb{F} G=\left\{\phi: G \rightarrow \mathcal{U}(\mathbb{F}) \mid \sum_{g \in G} \phi(g) \in \mathbb{F}\right\}
$$

together with the convolution product $(\phi * \psi)(g)=\sum_{g_{1} g_{2}=g} \phi\left(g_{1}\right) \psi\left(g_{2}\right)$ which is well-defined, because $\phi * \psi \in \mathbb{F} G$. These 3 -algebras quite often seem to be 3 -fields. For example, in the case where $G$ equals the additive group $\mathbb{Z} / n \mathbb{Z}$, we have $\mathbb{F} G=\mathbb{F}(n)$, as defined below. 
Definition 13 Fix a unital 3-field $\mathbb{F}$ and let

$$
\begin{aligned}
& \mathbb{F}\left[x_{1}, \ldots, x_{n}\right]= \\
& \left\{\sum_{|\alpha| \leq N} f_{\alpha} x^{\alpha} \mid \alpha=\left(\alpha_{1}, \ldots, \alpha_{n}\right), \quad N \in \mathbb{N}_{0}, \quad f_{\alpha} \in \mathcal{U}(\mathbb{F}), \quad \sum f_{\alpha} \in \mathbb{F}\right\} .
\end{aligned}
$$

We call this space the polynomial algebra in $n$ variables over the 3-field $\mathbb{F}$. This space is a unital 3-algebra under the usual product of polynomials.

We calculate that

$$
\begin{aligned}
& \mathcal{Q}\left(\mathbb{F}\left[x_{1}, \ldots, x_{n}\right]\right)=: \mathbb{F}\left[x_{1}, \ldots, x_{n}\right]^{\text {even }}= \\
& \left\{\sum_{|\alpha| \leq N} f_{\alpha} x^{\alpha} \mid \alpha=\left(\alpha_{1}, \ldots, \alpha_{n}\right), \quad N \in \mathbb{N}_{0}, \quad f_{\alpha} \in \mathcal{U}(\mathbb{F}), \quad \sum f_{\alpha} \in \mathcal{Q}(\mathbb{F})\right\} .
\end{aligned}
$$

and $\mathcal{U}\left(\mathbb{F}\left[x_{1}, \ldots, x_{n}\right]\right)=\mathcal{U}(\mathbb{F})\left[x_{1}, \ldots, x_{n}\right]$

Theorem 1 (Universality of polynomial algebras) The polynomial algebra $\mathbb{F}\left[x_{1}, \ldots, x_{n}\right]$ is universal in the class of unital 3 -algebras over $\mathbb{F}$, generated by $n$ elements.

The polynomial algebra $\mathbb{Q}^{\text {odd }}\left[x_{1}, \ldots, x_{n}\right]$ is universal in the class of all unital 3 -algebras over any of the prime fields, generated by $n$ elements.

\section{Proof}

1. If $A$ is an algebra generated by $a_{1}, \ldots, a_{n}$, define $\Psi: \mathbb{F}\left[x_{1}, \ldots, x_{n}\right] \rightarrow A$ by

$$
\Psi\left(\sum_{|\alpha| \leq N} f_{\alpha} x_{1}^{\alpha_{1}}, \ldots, x_{n}^{\alpha_{n}}\right)=\sum_{|\alpha| \leq N} f_{\alpha} a_{1}^{\alpha_{1}}, \ldots, a_{n}^{\alpha_{n}}
$$

and use Sect. 2 to see that $A \cong \mathbb{F}\left[x_{1}, \ldots, x_{n}\right] / \operatorname{ker} \Psi$.

2. The statement about $\mathbb{Q}^{\text {odd }}\left[x_{1}, \ldots, x_{n}\right]$ follows by applying the first part of this theorem for the respective prime field and then by combining the quotient mapping with the one from Example 12.

Example 17 Fix a unital 3-field $\mathbb{F}$ as well as a natural number $n$, and define

$$
\mathbb{F}(n)=\left\{P=f+\sum_{i=1}^{n-1} a_{i}(x-1)^{i} \mid a_{i} \in \mathcal{U}(\mathbb{F}), \quad f \in \mathbb{F}, \quad(x-1)^{n}=0\right\}
$$

Here, we use the language of 'generators and relations' so that requiring $(x-1)^{n}=$ 0 means that we divide out the ideal generated by this polynomial. It follows that 
$\mathbb{F}(n)$ is a unital 3-algebra, generated by the single polynomial $x-1$. It actually is a 3-field, as it is isomorphic to $\mathrm{T}(n, \mathbb{F})$, which we introduce in

Example 18 The Toeplitz field of order $n$ over $\mathbb{F}, \mathbb{T}(n, \mathbb{F})$, consists, as set, of all matrices

$$
t=\left(\begin{array}{cccc}
f & \cdots & 0 & 0 \\
b_{1} & \ddots & \ddots & 0 \\
\vdots & \ddots & \ddots & \vdots \\
b_{n-1} & \cdots & b_{1} & f
\end{array}\right), \quad f \in \mathbb{F}, \quad b_{i} \in \mathcal{U}(\mathbb{F}), \quad i=1, \ldots, n-1
$$

Note that the inverse of each $t$ is of the same form, and hence the Toeplitz fields are commutative 3-subfields of the triangular 3-fields from Example 26. The number of elements in this field is

$$
|\mathrm{T}(n, \mathbb{F})|=|\mathbb{F}||\mathcal{U}(\mathbb{F})|^{n-1}=2^{n-1}|\mathbb{F}|
$$

We now can show that $\mathbb{F}(n) \cong \mathbb{T}(n, \mathbb{F})$ (first, as ternary unital rings, and then, consequently, as fields): The 3 -vector space $\mathbb{F}(n)$ has basis

$$
E=\left\{e_{i}=(x-1)^{i} \mid i=0, \ldots, n-1\right\},
$$

and if we consider an element $P=f+\sum_{i=1}^{n-1} b_{i}(x-1)^{i} \in \mathbb{T}_{+}(n, \mathbb{F}), f \in$ $\mathbb{F}, \quad b_{i} \in \mathcal{U}(\mathbb{F}), i=1, \ldots, n-1$ as a linear map on $\mathbb{F}(n)$, then its matrix representation w.r.t. $E$ is given by

$$
t_{f}=\left(\begin{array}{cccc}
f & \cdots & 0 & 0 \\
b_{1} & \ddots & \ddots & 0 \\
\vdots & \ddots & \ddots & \vdots \\
b_{n-1} & \cdots & b_{1} & f
\end{array}\right)
$$

Since the product of $\mathbb{F}(n)$ turns out to be the matrix product of these matrices, the claim has been proven.

\section{Finite fields}

In general, the theory of the finite unital 3-fields looks quite different from the binary one, in which all such fields can be explicitly listed. In the case of unital 3-fields such a classification seems to be out of reach for the moment, which is due to the fact that the minimal number of generators for this type of field no longer has to be one, as in the binary case. In fact, finite unital 3-fields do not share much with their binary counterparts. 
Theorem 1 A finite unital 3-field $\mathbb{F}$ admits an embedding into a binary field $\mathbb{K}$ (equipped with its derived ternary addition) if and only if $\mathbb{F}=\{1\}$.

Proof If $n=\chi(\mathbb{F})>1$, then $\mathcal{Q}(\operatorname{Prim} \mathbb{F})=\left(\mathbb{Z} / 2^{n} \mathbb{Z}\right)^{\text {even }}$ is not an integral domain, and so Prim $\mathbb{F}$ (and much less $\mathbb{F}$ ) can be embedded into a binary field, in light of Theorem 4 . In case $\chi(\mathbb{F})=1$, we will see in Theorem 5 how a subfield of $\mathbb{F}$ looks which is generated by a single element, and the statement then follows as $\mathcal{Q}(\mathbb{F})$ in this case cannot be an integral domain, either.

Our second result remarkably pairs with the binary case, though.

Theorem 2 (Cardinality of finite fields) For each finite unital 3-field the number of elements is a power of 2 .

Proof Clearly, each finite unital 3-field $\mathrm{F}$ is a 3-vector space over PrimF. By Corollary 1 , the number of elements in $\mathbb{F}$ is $2^{n-1} \frac{|\operatorname{PrimF}|^{n}}{\left|\operatorname{ker} \phi_{\mathrm{F}}\right|}=2^{n-1} \frac{\chi(\mathbb{F})^{n}}{\left|\operatorname{ker} \phi_{\mathrm{F}}\right|}$. According to Theorem $2, \chi(\mathbb{F})$ is a power of 2 , which $\left|\operatorname{ker} \phi_{\mathbb{F}}\right|$ must divide, and the result follows.

For any polynomial $P=\sum_{v} a_{v} x^{v}$ in $\mathbb{Q}[x]$ we let $\|P\|_{2}=\max _{v}\left|a_{v}\right|_{2}$ (what some authors call the Gauss norm). Then $\|P\|_{2} \leq 1$ if and only if $P \in \mathcal{U}\left(\mathbb{Q}^{\text {odd }}\right)[x]$, and $\|P\|_{2}=1$ if, moreover, $P$ has at least one coefficient in $\mathbb{Q}^{\text {odd }}$. Then, for any $P, Q \in \mathbb{Q}[x]$, we have $\|P Q\|_{2}=\|P\|_{2}\|Q\|_{2}$. This follows from the fact that the product of two polynomials in $\mathbb{Z}[x]$, having both at least one odd coefficient, possesses itself at least one odd coefficient (see, for example, [3, pp. 35-43 ]).

The following Lemma follows from these properties of the Gauss norm.

Lemma 1 The irreducible polynomials for the ring $\mathcal{U}\left(\mathbb{Q}^{\text {odd }}\right)[x]$, i.e. those polynomials that cannot be factored into the product of two polynomials, are: the constant polynomial 2 and those polynomials $P$ which are irreducible in $\mathbb{Q}[x]$, and for which $\|P\|_{2}=1$.

Definition 14 Let $R$ be a unital 3-ring. We call $p \in \mathcal{Q}(R)$ completely even if and only if in every factorization of $p$ within $\mathcal{U}(R)$, factors are units of $R$ or elements from $\mathcal{Q}(R)$.

We will be concerned here with the polynomial ring $\mathbb{F}[x]$ in which a polynomial $P$ with coefficients in $\mathcal{U}(\mathbb{F})$ is completely even if and only if

1. $P$ belongs to $\mathbb{F}[x]^{\text {even }}$, and

2. $P$ factors only into odd factors which are units or even polynomials.

Theorem 3 Suppose $\mathbb{F}$ is a prime field and that $P_{0}$ is any polynomial in $\mathbb{F}[x]^{\text {even }}$. Then $\mathbb{F}[x] /\left\langle P_{0}\right\rangle$ is a unital 3-field if and only if $P_{0}$ is completely even.

Proof Clearly, whenever $P_{0}$ has a factorization $P_{0}=Q P$, with $Q$ a non-invertible odd polynomial, $\langle Q\rangle$ is an ideal larger than $\left\langle P_{0}\right\rangle$, strictly smaller than $\mathbb{F}[x]^{\text {even }}$, and intersecting $\mathbb{F}[x]^{\text {odd }}$. So $\mathbb{F}[x] /\left\langle P_{0}\right\rangle$ cannot be a 3 -field in light of Theorem 1 . 
For the converse, suppose $P_{0}$ is completely even. Additionally, we assume that $P_{0}$ does not contain any invertible odd factor, not affecting the following argument.

We first look at the case in which $|\mathbb{F}|=\infty$. For an ideal $\mathfrak{I} \in \mathcal{U}[x]$ we write $\mathfrak{I}_{\mathbb{Q}}$ for the ideal $\mathfrak{I}$ generates in $\mathbb{Q}[x]$, i.e.

$$
\mathfrak{I}_{\mathbb{Q}}=\left\{2^{-n} P \mid P \in \mathfrak{I}, \quad n \in \mathbb{N}\right\} .
$$

Since $\left(\mathcal{U}[x] P_{0}\right)_{\mathbb{Q}}$ is the principal ideal generated by $P_{0}$ in $\mathbb{Q}[x]$, we find for any ideal $\mathfrak{I}$ of (and different from) $\mathcal{U}[x]$, larger than $\mathcal{U}[x] P_{0}$, a factorization $P_{0}=P Q$ so that $\mathfrak{I}_{\mathbb{Q}}=\mathbb{Q}[x] P$. Since for no $n \in \mathbb{N}, 2^{-n}$ is a factor of $P_{0}$, we actually may suppose that $P, Q \in \mathcal{U}[x]$ and still have $\mathfrak{I}_{\mathbb{Q}}=\mathbb{Q}[x] P$. As $P_{0}$ was assumed to be completely even (and not containing any factor that is a unit) $P$ and $Q$ have to be even and so

$$
\mathfrak{I} \cap \mathcal{U}[x]^{\text {odd }} \subseteq \mathbb{Q}[x] P \cap \mathcal{U}[x]^{\text {odd }}=\varnothing
$$

proving that $\mathbb{F}[x] /\left\langle P_{0}\right\rangle$ is a field, by Theorem 1 .

Now suppose $|\mathbb{F}|=2^{n}$ and denote by

$$
\pi_{n}: \mathbb{Q}^{\text {odd }}[x] \rightarrow\left(\mathbb{Z} / 2^{n} \mathbb{Z}\right)^{\text {odd }}[x]
$$

the canonical quotient map, reducing the coefficients of elements in $\mathbb{Q}^{\text {odd }}[x]$ to coefficients in $\left(Z / 2^{n} Z\right)^{\text {odd }}$. We again will suppose that $P_{0}$ does not contain any invertible odd factor. Fix a polynomial $P_{1} \in \mathbb{Q}^{\text {even }}[x]$, of the same degree as $P_{0}$, such that $\pi_{n}\left(P_{1}\right)=P_{0}$. The map $\pi_{n}$ decreases degrees of polynmomials, preserving the one of $P_{1}$, hence any factorization of the latter polynomial into non-even factors would result into one of the same kind for $P_{0}$. Therefore $P_{1}$ has to be completely even as well, and so by the first part of the proof, $\mathbb{Q}^{\text {odd }}[x] /\left\langle P_{1}\right\rangle$ is a unital 3-field. The result then follows from the fact that for $|\mathbb{F}|=2^{n}$

$$
\mathbb{F}[x] /\left\langle P_{0}\right\rangle=\pi_{n}\left(\mathbb{Q}^{\text {odd }}[x] /\left\langle P_{1}\right\rangle\right) .
$$

Corollary 2 Each finite, singly generated unital 3-field $\mathrm{F}$ is the quotient of a singly generated unital 3-field $\widehat{\mathbb{F}}$ with prime field $\mathbb{Q}^{\text {odd }}$.

Factoring polynomials is no easy business under these circumstances. For the example below, we will use

Lemma 2 Let $R$ be a unital 3-ring and $p \in \mathcal{Q}(R)$. If $\phi: R \rightarrow R^{\prime}$ is a morphism respecting non-units and $\phi(p)$ is non-zero and completely even then $p$ is completely even.

Proof The proof is easy: If there is a factorization of $p$ in which one of the factors is odd and not a unit, it follows that the same type of factorization exists for $\phi(p)$, as $\phi$ respects (non-units and) the distinction between even and odd. 
Example 19 Let $\mathbb{F}$ be any of the prime 3-fields and denote by $\phi: \mathbb{F}[x] \rightarrow \mathbb{F}_{0}[x]$ the morphism reducing coefficients $\bmod 2$. We claim that $\phi$ is a morphism as in the lemma:

Recall that in $R[x], R$ a unital (binary) ring, $P=a_{0}+\ldots+a_{n} x^{n}$ is a unit if $a_{0}$ is a unit in $R$ and $a_{v}$ is nilpotent for all $v \geq 1$. For a non-unit $P \in \mathbb{F}[x]$ then, either $a_{0}$ is even, in which case it is mapped to a non-unit in $\mathbb{F}_{0}$, or, $a_{0}$ is a unit but one of the coefficients, $a_{k}, k>1$, is mapped to 1 , and so also in this case $\phi(P)$ is a nonunit.

Example 20 Let $\mathbb{F}$ be any of the prime 3-fields. Then $x^{n}-1$ is completely even if $n$ is a power of two. In fact, let $n=n_{0} 2^{k}$ with $n_{0}$ odd. Then

$$
\begin{aligned}
& x^{n}-1=\left(x^{n_{0}}-1\right)\left(x^{n_{0}}+1\right)\left(x^{2 n_{0}}+1\right) \cdots\left(x^{n_{0} 2^{k-1}}+1\right)=x \\
& =(x-1)\left(\sum_{m=0}^{n_{0}-1} x^{m}\right) \prod_{j=0}^{k-1}\left(x^{2^{j} n_{0}}+1\right) .
\end{aligned}
$$

If $n_{0}>1$, the polynomial $\sum_{m=0}^{n_{0}-1} x^{m}$ is odd (and not a unit), so $x^{n}-1$ is not completely even. If, however, $n_{0}=1$ we invoke the morphism $\phi$ from above that reduces coefficients to the (binary) field $\mathbb{Z} / 2$. Using the Frobenius morphism, for $n$ a power of 2 , we have $x^{n}-1=(x-1)^{n}$, and the claim follows.

We collect the above into

Theorem 4 Let $\mathbb{F}$ be any of the finite prime fields. Then, there is a splitting unital 3 -field for $\mathbb{F}$ and the polynomial $x^{n}-1$ if and only if $n$ is a power of 2 .

Note that in classical (binary) field theory there is a splitting field for the finite prime fields (essentially) for all the polynomials $x^{n}-1$.

Finally, we will consider the already quite difficult case of finite unital 3-fields for which the characteristic is one, $\chi(\mathbb{F})=1$.

Theorem 5 Suppose $\mathbb{F}$ is a finite unital 3-field with $\chi(\mathbb{F})=1$, and denote by $\mathbb{F}_{0}=\{1\}$ its prime field. If $\mathbb{F}$ is generated by a single element, then it is isomorphic to $\mathbb{F}_{0}[x] /\left\langle(x-1)^{n}\right\rangle$. Furthermore,

$$
\mathbb{F}_{0}[x] /\left\langle(x-1)^{n}\right\rangle \cong \mathbb{F}_{0}(n),
$$

where $\mathbb{F}_{0}(n)$ was defined in Example 17.

Proof By the above result, and since $\mathcal{U}\left(\mathbb{F}_{0}\right)=\mathbb{Z} / 2 \mathbb{Z}$ is a (binary) field, there must be a completely even polynomial $P \in(\mathbb{Z} / 2 \mathbb{Z})[x]$ so that $\mathbb{F}=\mathbb{F}_{0}[x] /\langle P\rangle$. Writing a polynomial $Q \in(\mathbb{Z} / 2 \mathbb{Z})[x]$ in powers of $(x-1)$ we have that $Q$ is even if its constant term is even, and it is odd if its constant term is. Now, if the polynomial $P$ is not of the desired form, there are $n_{0}<\ldots<n_{k}$ with

$$
P=(x-1)^{n_{0}}+\ldots+(x-1)^{n_{k}}=(x-1)^{n_{0}}\left(1+\ldots+(x-1)^{n_{k}-n_{0}}\right) .
$$


Hence, such a polynomial is not completely even, and we are done.

Finally, $\mathbb{F}_{0}[x] /\left\langle(x-1)^{n}\right\rangle \cong \mathbb{F}_{0}(n)$ is proven as follows. Consider the mapping $\Phi: \mathbb{F}_{0}[x] \longrightarrow \mathbb{F}_{0}(n)$,

$$
\Phi(Q)=\left(Q(1), \ldots, \frac{Q^{(v)}(1)}{\nu !}, \ldots, \frac{Q^{(n-1)}(1)}{(n-1) !}\right) \bmod 2
$$

where only the coefficients of the respective polynomials in $\mathbb{F}_{0}(n)$ are written down and (formal) derivatives are used. This map is a (surjective) field morphism: it clearly is additive, while multiplicativity follows as the product of Taylor polynomials of two given polynomials also in the present situation is the Taylor polynomial of the their products. Since the kernel of this map is equal to $\left\langle(x-1)^{n}\right\rangle$, the second statement of the theorem follows.

We look into some further examples and determine some automorphism groups.

Lemma 3 There is a 1-1 correspondence between the automorphisms of $\mathbb{F}_{0}(n)$ and polynomials $P=\sum_{j} f_{j} x^{j}, f_{j} \in \mathcal{U}\left(\mathbb{F}_{0}(n)\right), \sum f_{j} \in \mathbb{F}_{0}$, for which there exists another such polynomial $Q$ with $P \circ Q=Q \circ P=x$.

Proof Suppose $\Psi$ is an automorphism of $\mathbb{F}_{0}(n)$. Let $P=\Psi(x)=\sum_{j} f_{j} x^{j}$. Then $f_{j} \in \mathcal{U}\left(\mathbb{F}_{0}(n)\right)$ and $\sum f_{j} \in \mathbb{F}_{0}$. Since the polynomial $x$ is generating, $P$ uniquely determines $\Psi$. Furthermore, $\Psi\left(\sum_{j} g_{j} x^{j}\right)=\sum_{j} g_{j} P^{j}$, and so composition of morphisms consists in substitution of polynomials. In particular, $\Psi^{-1}$ must correspond to a polynomial $Q$ with $P \circ Q=Q \circ P=x$.

Example 21 Let us start with a classical field extension. For $\chi(\mathbb{F})=1$ we formally adjoin a square root of 3 and obtain $\mathbb{F}[\sqrt{3}]$. More explicitly,

$$
\mathbb{F}[\sqrt{3}]=\{a+b \sqrt{3} \mid a, b \in \mathbb{Z} / 2 \mathbb{Z}, a+b=1\},
$$

and it turns out that this 3-field is isomorphic to $\mathbb{F}(2)$. Note that this field has a trivial automorphism group.

Example 22 Let us have a look at $\mathbb{F}(3)$, where $\mathbb{F}$ is as before. We use polynomials in the generator $x$ and denote the elements $a=x, b=x^{2}, c=x^{2}+x+1$, $a, b, c, 1 \in \mathbb{F}$ (3). The multiplicative Cayley table is

\begin{tabular}{c|c|c|c|c}
$\cdot$ & 1 & $a$ & $b$ & $c$ \\
\hline 1 & 1 & $a$ & $b$ & $c$ \\
\hline$a$ & $a$ & $b$ & $c$ & 1 \\
\hline$b$ & $b$ & $c$ & 1 & $a$ \\
\hline$c$ & $c$ & 1 & $a$ & $b$
\end{tabular}


To find nontrivial automorphisms on $\mathbb{F}$ (3) we construct the Cayley table under compositions as

\begin{tabular}{c|c|c|c}
$\circ$ & $a$ & $b$ & $c$ \\
\hline$a$ & $a$ & $b$ & $c$ \\
\hline$b$ & $b$ & 1 & $b$ \\
\hline$c$ & $c$ & $b$ & $a=x$
\end{tabular}

We observe that one nontrivial automorphism on $\mathbb{F}(3)$ is connected to $c$, because $c \circ c=a=x$. So the group of automorphisms is $\mathbb{Z} / 2 \mathbb{Z}$, or, for later use, the dihedral group of order 2 , respectively.

Example 23 Let us denote elements of F (4) as

$$
\begin{array}{c|c|c|c|c|c|c}
a & b & c & d & e & f & g \\
\hline x & x^{2} & x^{3} & x^{2}+x+1 & x^{3}+x+1 & x^{3}+x^{2}+1 & x^{3}+x^{2}+x
\end{array}
$$

The multiplicative Cayley table for $\mathbb{F}(4)$ is

\begin{tabular}{c|llllllll}
$\cdot$ & 1 & $a$ & $b$ & $c$ & $d$ & $e$ & $f$ & $g$ \\
\hline 1 & 1 & $a$ & $b$ & $c$ & $d$ & $e$ & $f$ & $g$ \\
\hline$a$ & $a$ & $b$ & $c$ & 1 & $g$ & $d$ & $e$ & $f$ \\
\hline$b$ & $b$ & $c$ & 1 & $a$ & $f$ & $g$ & $d$ & $e$ \\
\hline$c$ & $c$ & 1 & $a$ & $b$ & $e$ & $f$ & $g$ & $d$ \\
\hline$d$ & $d$ & $g$ & $f$ & $e$ & $b$ & $a$ & 1 & $c$ \\
\hline$e$ & $e$ & $d$ & $g$ & $f$ & $a$ & 1 & $c$ & $b$ \\
\hline$f$ & $f$ & $e$ & $d$ & $g$ & 1 & $c$ & $b$ & $a$ \\
\hline$g$ & $g$ & $f$ & $e$ & $d$ & $c$ & $b$ & $a$ & 1
\end{tabular}

To find nontrivial automorphisms we make use of the Cayley table w.r.t. composition, and we find that the (nontrivial) automorphisms are exactly those given by the polynomials $c, d, f$. Composition is then given by the following table

\begin{tabular}{c|cccc}
$\circ$ & $a$ & $c$ & $d$ & $f$ \\
\hline$a$ & $a=x$ & $c$ & $d$ & $f$ \\
\hline$c$ & $c$ & $a=x$ & $f$ & $d$ \\
\hline$d$ & $d$ & $f$ & $a=x$ & $c$ \\
\hline$f$ & $f$ & $d$ & $c$ & $a=x$
\end{tabular}

which upon inspection yields that the automorphism group equals the dihedral group of order 4 . 
Example 24 Let us denote elements of F (5) as

\begin{tabular}{c|c|c|c|c|c|c}
$a$ & $b$ & $c$ & $d$ & $e$ & $f$ & $g$ \\
\hline$x$ & $x^{2}$ & $x^{3}$ & $x^{4}$ & $x^{2}+x+1$ & $x^{3}+x+1$ & $x^{4}+x+1$ \\
\multicolumn{2}{c|}{$p$} & $q$ & $r$ & $s$ \\
\hline$x^{3}+x^{2}+1$ & $x^{4}+x^{2}+1$ & $x^{4}+x^{3}+1$ & $x^{3}+x^{2}+x$ \\
\multicolumn{1}{c|}{$t$} & $u$ & $v$ & $w$ \\
\hline$x^{4}+x^{2}+x$ & $x^{4}+x^{3}+x$ & $x^{4}+x^{3}+x^{2}$ & $x^{4}+x^{3}+x^{2}+x+1$
\end{tabular}

Then similar calculations give us the nontrivial automorphisms induced by the polynomials $c, e, g, p, r, t, v$. Together with the identity morphism $a=x$ they obey the following Cayley table under composition

\begin{tabular}{c|cccccccc}
$\circ$ & $a$ & $c$ & $e$ & $g$ & $p$ & $r$ & $t$ & $v$ \\
\hline$a$ & $a=x$ & $c$ & $e$ & $g$ & $p$ & $r$ & $t$ & $v$ \\
\hline$c$ & $c$ & $a=x$ & $p$ & $r$ & $e$ & $g$ & $v$ & $t$ \\
\hline$e$ & $e$ & $v$ & $g$ & $t$ & $c$ & $p$ & $a=x$ & $r$ \\
\hline$g$ & $g$ & $r$ & $t$ & $a=x$ & $v$ & $c$ & $e$ & $p$ \\
\hline$p$ & $p$ & $t$ & $r$ & $v$ & $a=x$ & $e$ & $c$ & $g$ \\
\hline$r$ & $r$ & $g$ & $v$ & $c$ & $t$ & $a=x$ & $p$ & $e$ \\
\hline$t$ & $t$ & $p$ & $a=x$ & $e$ & $r$ & $v$ & $g$ & $c$ \\
\hline$v$ & $v$ & $e$ & $c$ & $p$ & $g$ & $t$ & $r$ & $a=x$
\end{tabular}

This is the dihedral group of order 8 .

Note that all these automorphism groups would be the "Galois groups" for the respective extensions of $\{1\}$. Also, $\mathbb{F}(n)$ is a quotient of $\mathbb{F}(m)$ whenever $n<m$.

The class of all unital 3-field of characteristic different from one might be inaccessible for a (concrete) classification. Here are some examples, where the respective fields require more than one generator in the present definition.

Fix a unital 3-field $\mathbb{F}$, and put

$$
\mathbb{F}_{0}\left(n_{1}, \ldots, n_{k}\right)=\mathbb{F}_{0}\left[x_{1}, \ldots, x_{k}\right] /\left\langle\left(x_{1}-1\right)^{n_{1}}, \ldots,\left(x_{k}-1\right)^{n_{k}}\right\rangle
$$

This extension of $\mathbb{F}$ is characterized by the fact that it displays the fewest possible relations a field of $k$ generators possibly can have (this will be made precise below). It can be shown that

$$
\begin{aligned}
& \mathbb{F}_{0}\left(n_{1}, \ldots, n_{k}\right)= \\
& \left\{1+\sum_{(1, \ldots, 1) \leq \alpha \leq\left(n_{1}-1, \ldots, n_{k}-1\right)} \varepsilon_{\alpha}(1-x)^{\alpha} \mid \varepsilon_{\alpha}=0,1, \quad\left(x_{k}-1\right)^{n_{k}}=0, k=1, \ldots, n\right\},
\end{aligned}
$$

where $(1-x)^{\alpha}=\left(1-x_{1}\right)^{\alpha_{1}} \ldots\left(1-x_{n}\right)^{\alpha_{n}}$. Much more relations are necessary in order to present the Cartesian product $\mathbb{F}_{0}\left(n_{1}\right) \times \ldots \times \mathbb{F}_{0}\left(n_{k}\right)$. Denote by $\xi_{i}$ the generator of the 3 -field $\mathbb{F}_{0}\left(n_{i}\right)$ and by $x_{i}$ the element of $\mathbb{F}_{0}\left(n_{1}\right) \times \ldots \times \mathbb{F}_{0}\left(n_{k}\right)$ which has the 
unit element in each entry except at the place $i$ where it is $\xi_{i}$. Then for each element $\left(f_{1}, \ldots, f_{n}\right) \in \mathbb{F}_{0}\left(n_{1}\right) \times \ldots \times \mathbb{F}_{0}\left(n_{k}\right)$ there are $\varepsilon_{i j}=0,1$ so that

$$
\left(f_{1}, \ldots, f_{n}\right)=(1, \ldots, 1)+\sum_{i=1}^{n} \sum_{j=1}^{k_{n}} \varepsilon_{i j}\left(1-x_{i}\right)^{j} .
$$

Consequently,

$$
\begin{aligned}
& \mathbb{F}_{0}\left(n_{1}\right) \times \ldots \times \mathbb{F}_{0}\left(n_{k}\right)=\mathbb{F}_{0}\left[x_{1}, \ldots, x_{k}\right] / \\
& \left\langle\left(x_{1}-1\right)^{n_{1}}, \ldots,\left(x_{k}-1\right)^{n_{k}},\left(x_{i}-1\right)\left(x_{j}-1\right), i, j=1, \ldots, n, i \neq j\right\rangle
\end{aligned}
$$

Theorem 6 Let $\mathbb{F}$ be a finite field with $\chi(\mathbb{F})=1$, generated as a unital 3-field by $n$ elements. Let, as before, $\mathbb{F}_{0}=(\mathbb{Z} / 2 \mathbb{Z})^{\text {odd }}=\{1\}$.

1. There exist natural numbers $k_{1}, \ldots, k_{n}$ such that $\mathbb{F}$ is a quotient of $\mathbb{F}_{0}\left(k_{1}, \ldots, k_{n}\right)$.

2. The ideal $\mathcal{J}$ such that $\mathbb{F} \cong \mathbb{F}_{0}\left[x_{1}, \ldots, x_{n}\right] / \mathcal{J}$ is of the form

$$
\mathcal{J}=\left\langle\left(x_{1}-1\right)^{k_{1}}, \ldots,\left(x_{n}-1\right)^{k_{n}}, P_{1}, \ldots, P_{N}\right\rangle \text {, }
$$

where the polynomials $P_{1}, \ldots, P_{N}$ are neither divisible by an odd polynomial nor by any of the $\left(x_{k}-1\right)^{n_{k}}$.

Proof Denote by $x_{1}, \ldots, x_{n}$ the 3-field generators of $\mathbb{F}$. As each of them generates a unital 3-field, $\left(x_{i}-1\right)^{k_{i}}=0$ for some $k_{i}, i=1, \ldots, n$, and it follows that there is a quotient map of $\mathbb{F}_{0}\left(k_{1}, \ldots, k_{n}\right)$ onto $\mathbb{F}$.

In order to prove the second part of the theorem, we select even polynomials $P_{1}, \ldots, P_{N}$, not divisible by any of the $\left(x_{k}-1\right)^{n_{k}}$, so that $\mathbb{F} \cong \mathbb{F}_{0}\left(k_{1}, \ldots, k_{n}\right) /$ $\left\langle P_{1}, \ldots, P_{N}\right\rangle$. Similar to the proof of Theorem 5, one can show that it is not possible that any of these polynomials contains an odd factor (Alternatively, one can use the fact that all odd polynomials which can arise as factors here are invertible.)

Example 25 Let us consider the "unfree" unital 3-field $\mathbb{F}^{2}$ and the "free" unital 3-field $\mathbb{F}\left(n_{1}, n_{2}\right)$. We show that $\mathbb{F}(2) \times \mathbb{F}(2)$ and $\mathbb{F}(2,2)$ are not isomorphic. By definition, we have $\mathbb{F}(2)=\{1+\varepsilon(y-1) \mid \varepsilon \in \mathbb{Z} / 2 \mathbb{Z}\}$, which contains 2 elements $\{1, y\}$ with the relations (as pairs) $1+1=y+y=0$ and $y^{2}=1$. The most unfree 3 -field $\mathbb{F}(2) \times \mathbb{F}(2)$ has 4 elements and generated by $x_{1}=\left(\begin{array}{l}y \\ 1\end{array}\right), x_{2}=\left(\begin{array}{l}1 \\ y\end{array}\right)$. It is easily seen that $x_{1}^{2}=x_{2}^{2}=1, x_{1} x_{2}=x_{1}+x_{2}-1$, and therefore

$$
\mathbb{F}(2) \times \mathbb{F}(2)=\mathbb{Z}_{2}\left[x_{1}, x_{2}\right]^{\text {odd }} /\left\langle\left(x_{1}-1\right)^{2},\left(x_{2}-1\right)^{2}, P\right\rangle,
$$


where the additional polynomial is $P=\left(x_{1}-1\right)\left(x_{2}-1\right)$ (see (62)). On the other hand,

$$
\mathbb{F}(2,2)=\left\{1+\varepsilon_{1}\left(x_{1}-1\right)+\varepsilon_{2}\left(x_{2}-1\right)+\varepsilon\left(x_{1}-1\right)\left(x_{2}-1\right) \mid \varepsilon_{i} \in \mathbb{Z}_{2}\right\}
$$

contains 8 elements and

$$
\mathbb{F}(2,2)=\mathbb{Z} / 2 \mathbb{Z}\left[x_{1}, x_{2}\right]^{\text {odd }} /\left\langle\left(x_{1}-1\right)^{2},\left(x_{2}-1\right)^{2}\right\rangle,
$$

which is not isomorphic to the field in (63).

Our final examples show that there exist noncommutative finite unital 3-fields.

Example 26 (Triangular 3-fields) Let $\mathbb{F}$ be a unital 3-field and put

$$
\mathbb{D}(n, \mathbb{F})=\left\{A_{n}\left(f_{i}, b_{i j}\right) \mid f_{i} \in \mathbb{F}, b_{i j} \in \mathcal{U}(\mathbb{F}), i . j=1, \ldots, n\right\},
$$

where

$$
A_{n}\left(f_{i}, b_{i j}\right)=\left(\begin{array}{ccc}
f_{1} & & 0 \\
& \ddots & \\
b_{i j} & & f_{n}
\end{array}\right)
$$

In order to see that $\mathrm{D}(n, \mathbb{F})$ is a unital 3-field, observe that sums and products of two such matrices lie again in $\mathrm{D}(n, \mathbb{F})$ and $\mathrm{D}(n, \mathbb{F})$ is a noncommutative unital 3-algebra (if $n>1$ ). Furthermore, each matrix (67) is invertible, and its inverse has the form

$$
A_{n}\left(f_{i}, b_{i j}\right)^{-1}=A_{n}\left(f_{i}^{-1}, \widehat{b}_{i j}\right)=\left(\begin{array}{ccc}
f_{1}^{-1} & & 0 \\
& \ddots & \\
\widehat{b}_{i j} & & f_{n}^{-1}
\end{array}\right),
$$

where $\widehat{b}_{i j} \in \mathcal{U}(\mathbb{F})$. This follows from the fact that the standard procedure for the inversion of a matrix yields expressions which are well-defined within $\mathbb{F}$. In total, $\mathrm{D}(n, \mathbb{F})$ is a noncommutative unital 3-field, which is finite in case $\mathbb{F}$ is.

Example 27 (Quaternion 3-fields) We start by selecting a unital 3-field F, and will equip the free 3 -vector space (with basis elements $i_{\mu}, \mu=0,1,2,3$ )

$$
\mathbb{H F}=\left(\mathbb{F}^{4}\right)^{\text {free }}=\left\{\sum_{\mu=0}^{3} a_{\mu} i_{\mu} \mid a_{\mu} \in \mathcal{U}(\mathbb{F}), \sum_{\mu=0}^{3} a_{\mu} \in \mathbb{F}\right\},
$$


with a multiplication (so that it will become a unital 3-field) in the following way: We assume the quaternion relations

$$
i_{1}^{2}=i_{2}^{2}=i_{3}^{2}=i_{1} i_{2} i_{3}=-1, i_{0}=1
$$

and linearly extend them to a multiplication on the whole of $\mathrm{HF}$. This product is well-defined, since the sum of coefficients $c_{k} \in \mathcal{U}(\mathbb{F})$ in

$$
\left(\sum_{k=0}^{3} a_{k} i_{k}\right)\left(\sum_{k=0}^{3} b_{k} i_{k}\right)=\sum_{k=0}^{3} c_{k} i_{k},
$$

where $a_{k}, b_{k} \in \mathcal{U}(\mathbb{F}), \sum_{k=0}^{3} a_{k} \in \mathbb{F}, \sum_{k=0}^{3} b_{k} \in \mathbb{F}$ can be written in the form

$$
\sum_{k=0}^{3} c_{k}=\left(\sum_{k=0}^{3} a_{k}\right)\left(\sum_{k=0}^{3} b_{k}\right)-2 \gamma, \quad \gamma \in \mathcal{U}(\mathbb{F}) .
$$

Since, in general, for an element $u \in \mathcal{U}(\mathbb{F})$, we have that $2 u \in \mathcal{Q}(\mathbb{F})$, it follows that the sum over the $c_{k}$ 's is in $\mathbb{F}$, and $\mathrm{HF}$ is a unital 3-algebra. In addition, if $q=a_{0}+a_{1} i_{1}+a_{2} i_{2}+a_{3} i_{3}$, we let $\bar{q}=a_{0}-a_{1} i_{1}-a_{2} i_{2}-a_{3} i_{3}$ and observe that $q \bar{q}=\left(a_{0}^{2}+a_{1}^{2}+a_{2}^{2}+a_{3}^{2}\right) i_{0} \in \mathbb{H F}$. Then, for each element $\mathrm{q}$ of $\mathrm{HF}$, an inverse is given by

$$
q^{-1}=\frac{\bar{q}}{q \bar{q}}=\frac{a_{0}-a_{1} i_{1}-a_{2} i_{2}-a_{3} i_{3}}{a_{0}^{2}+a_{1}^{2}+a_{2}^{2}+a_{3}^{2}} \in \mathbb{H F}
$$

and thus, $\mathrm{HF}$ is a non-commutative 3 -field. (In this construction, the elements $i_{k}$ can be replaced by the canonical generators/relations of Clifford algebras in order to obtain a wider class of examples.)

Why is the above construction not possible if $\mathrm{F}$ is replaced by a finite binary field? The main reason is that in ternary fields there are no zero elements. In fact, each finite binary field, for some prime $p, \mathbb{Z} / p$ is a subfield, and now the probably fastest way to find an obstruction is through Lagrange's four-square theorem, by which each integer, hence also the prime $p$, is the sum of four squares.

Acknowledgements Research supported by the ERC through AdG 267079, as well as by Deutsche Forschungsgemeinschaft (DFG) within the framework of Exzellenzstrategie des Bundes und der Länder EXC 2044-390685587, Mathematik Münster: Dynamik -- Geometrie -- Struktur.

The first author (S.D.) would like to express his gratitude to Joachim Cuntz and Raimar Wulkenhaar for financial support, at a very early stage of this work.

Funding Open Access funding provided by Projekt DEAL.

Open Access This article is licensed under a Creative Commons Attribution 4.0 International License, which permits use, sharing, adaptation, distribution and reproduction in any medium or format, as long as you give appropriate credit to the original author(s) and the source, provide a link to the Creative Commons licence, and indicate if changes were made. The images or other third party material in this article are included in the article's Creative Commons licence, unless indicated otherwise in a credit line to the material. If material is not included in the article's Creative Commons licence and your intended use is not permitted by statutory regulation or exceeds the permitted use, you will need to obtain permission directly 
from the copyright holder. To view a copy of this licence, visit http://creativecommons.org/licenses/by/4. $0 /$.

\section{References}

1. Bagger, J. and N. Lambert [2008]. Gauge symmetry and supersymmetry of multiple M2-branes. Phys. Rev. D77, 065008.

2. Bohle, D. and W. Werner [2015]. A K-theoretic approach to the classification of symmetric spaces. J. Pure and App. Algebra 219 (10), 4295-4321.

3. Bosch, S., Guentzer, U., and Remmert, R. [1984]. Non-Archimedean Analysis, Grundlehren der mathematischen Wissenschaften 261. Springer.

4. Celakoski, N. [1977]. On $(F, G)$-rings. God. Zb., Mat. Fak. Univ. Kiril Metodij Skopje 28, 5-15.

5. Chu, C.-H. [2012]. Jordan structures in geometry and analysis, Volume 190 of Cambridge Tracts in Mathematics. Cambridge: Cambridge University Press.

6. Crombez, G. [1973]. The Post coset theorem for $(n, m)$-rings. Ist. Veneto Sci. Lett. Arti, Atti, Cl. Sci. Mat. Natur. 131, 1-7.

7. Crombez, G. and J. Timm [1972]. On (n,m)-quotient rings. Abh. Math. Semin. Univ. Hamb. 37, 200-203.

8. Čupona, G. [1965]. On [m,n]-rings. Bull. Soc. Math. Phys. Macedoine 16, 5-9.

9. de Azcarraga, J. A. and J. M. Izquierdo [2010]. n-Ary algebras: A review with applications. J. Phys. A43, 293001.

10. Dörnte, W. [1929]. Unterschungen über einen verallgemeinerten Gruppenbegriff. Math. Z. 29, 1-19.

11. Duplij, S. [2001]. Ternary Hopf algebras. In: A. G. Nikitin and V. M. Boyko (Eds.), Symmetry in Nonlinear Mathematical Physics, Kiev: Institute of Mathematics, pp. 25-34.

12. Elgendy, H. A. and M. R. Bremner [2012]. Universal associative envelopes of ( $n+1)$-dimensional $n$-Lie algebras. Comm. Algebra 40 (5), 1827-1842.

13. Higgins, P. J. [1956]. Groups with multiple operators. Proc. London Math. Soc. (3) 6, 367-416.

14. Jacobson, N. [1989]. Basic Algebra II, New York: Freeman and Company

15. Kerner, R. [2000]. Ternary algebraic structures and their applications in physics, preprint Univ. P. \& M. Curie, Paris, 15 p.

16. Kurosh, A. G. [1969]. Multioperator rings and algebras. Russian Math. Surveys 24, 1-13.

17. Leeson, J. J. and A. T. Butson [1980]. On the general theory of (m,n) rings. Algebra Univers. 11, 42-76.

18. Nambu, Y. [1973]. Generalized Hamiltonian dynamics. Phys. Rev. D7, 2405-2412.

19. Post, E. L. [1940]. Polyadic groups. Trans. Amer. Math. Soc. 48, 208-350.

20. Upmeier, H. [1985]. Symmetric Banach manifolds and Jordan $C^{*}$-algebras, Volume 104 of NorthHolland Mathematics Studies. Amsterdam: North-Holland Publishing. 